\title{
Gametocyte carriage after seasonal malaria chemoprevention in Plasmodium falciparum infected asymptomatic children
}

\author{
Abdullahi Ahmad ${ }^{1,3^{*}} \mathbb{D}$, Aurelia Prom ${ }^{1}$, John Bradley², Mamadou Ndiath', Blessed Etoketim', Mamadou Bah', \\ Jean-Pierre Van Geertruyden ${ }^{3}$, Chris Drakeley ${ }^{4}$, Teun Bousema ${ }^{5}$, Jane Achan ${ }^{1}$ and Umberto D'Alessandro ${ }^{1}$
}

\begin{abstract}
Background: Treatment of clinical Plasmodium falciparum malaria with sulfadoxine-pyrimethamine (SP) and amodiaquine (AQ) is associated with increased post-treatment gametocyte carriage. The effect of seasonal malaria chemoprevention (SMC) with SP and $\mathrm{AQ}$ on gametocyte carriage was assessed in asymptomatic $P$. falciparum infected children.
\end{abstract}

Methods: The study was carried out in eastern Gambia. Asymptomatic P. falciparum malaria infected children aged 24-59 months old who were eligible to receive SMC (SMC group) and children 5-8 years that were not eligible to receive SMC (comparison group) were recruited. Gametocytaemia was determined by molecular methods before and after SMC administration. Gametocyte carriage between the groups was compared using the chi-squared test and within-person using conditional logistic regression.

Results: During the 2017 and 2018 malaria transmission seasons, 65 and 75 children were recruited in the SMC and comparison groups, respectively. Before SMC administration, gametocyte prevalence was $10.7 \%(7 / 65)$ in the SMC group and $13.3 \%(10 / 75)$ in the comparison group $(p=0.64)$. At day $13(I Q R 12,13)$ after SMC administration, this was $9.4 \%(5 / 53)$ in children who received at least the first dose of SMC treatment and 12.7\% (9/71) for those in the comparison group $(p=0.57)$. Similarly, there was no difference in prevalence of gametocytes between children that adhered to all 3-day doses of SMC treatment $15.6 \%(5 / 32)$ and those in the comparison group $(p=0.68)$. In the SMC group, within-group gametocyte carriage was similar before and after SMC administration in children that received at least the first dose of SMC treatment $(\mathrm{OR} 0.6,95 \% \mathrm{Cl} 0.14-2.51 ; p=0.48)$ and in those that adhered to all 3-day doses of SMC treatment (OR 1.0, 95\% $\mathrm{Cl} 0.20-4.95 ; p=1.0)$.

Conclusion: In this study with relative low gametocyte prevalence prior to SMC treatment, no evidence was observed that SMC treatment increased gametocyte carriage in asymptomatic P. falciparum malaria infected children.

Keywords: Gametocyte carriage, Seasonal malaria chemoprevention, Plasmodium falciparum, Asymptomatic

*Correspondence: aahmad@mrc.gm; abdulbag@yahoo.com ${ }^{1}$ Disease Control and Elimination Theme, Medical Research Council Unit The Gambia At London, School of Hygiene and Tropical Medicine, P.O Box 273, Banjul, The Gambia

Full list of author information is available at the end of the article

\section{Background}

The global malaria burden has substantially reduced over the last two decades owing to the scale up of control interventions $[1,2]$. In sub-Saharan Africa, Plasmodium falciparum is the predominant malaria species and remains the focus of control and elimination efforts [2]. Since 2012, the World Health Organization (WHO)

(c) The Author(s) 2021. This article is licensed under a Creative Commons Attribution 4.0 International License, which permits use, sharing, adaptation, distribution and reproduction in any medium or format, as long as you give appropriate credit to the original author(s) and the source, provide a link to the Creative Commons licence, and indicate if changes were made. The images or other third party material in this article are included in the article's Creative Commons licence, unless indicated otherwise in a credit line to the material. If material is not included in the article's Creative Commons licence and your intended use is not permitted by statutory regulation or exceeds the permitted use, you will need to obtain permission directly from the copyright holder. To view a copy of this licence, visit http://creativeco mmons.org/licenses/by/4.0/. The Creative Commons Public Domain Dedication waiver (http://creativecommons.org/publicdomain/ zero/1.0/) applies to the data made available in this article, unless otherwise stated in a credit line to the data. 
recommends seasonal malaria chemoprevention (SMC) as an additional tool for malaria control where transmission is highly seasonal, primarily the Sahel region of sub-Saharan Africa [3]. SMC is the monthly administration of a full course of sulfadoxine-pyrimethamine (SP) and amodiaquine (AQ) to all children 3-59 months old during the 3-4 months of the malaria transmission season regardless of infection status with the goal of reducing malaria morbidity and mortality.

Given that many $P$. falciparum infections are asymptomatic in both high and low transmission settings [4, $5]$ and can persist for long periods of time [4, 6, 7], the systematic administration of anti-malarial drugs to the whole population or to high risk groups, such as children, results in the treatment of such infections. Most available anti-malarial treatments clear asexual parasite stages but with incomplete and variable effects on gametocytes [8], the sexual stages responsible for onward transmission to the vector. Treatment of symptomatic patients with $\mathrm{SP}, \mathrm{AQ}$, chloroquine $(\mathrm{CQ})$, and piperaquine $(\mathrm{PQ})$ is associated with increased gametocyte carriage and or density [9-14]. Mechanisms such as enhancement of gametocyte production in response to drug-induced stress, release of sequestered gametocytes and up-regulation of gametocyte production in response to subcurative dosage are current hypotheses for the emergence of post-treatment gametocytes [14, $15]$, that can be infectious to the vector $[12-14,16]$. Although the transmission potential of post-treatment gametocytes has been known for about 100 years [17], it has only recently drawn attention in relation to the current drive towards malaria elimination and eradication and the increased knowledge on the differential impact of anti-malarials on gametocyte persistence and infectivity [18].

SMC consists of the monthly administration of SP and AQ over 3 days. Only the first day of treatment (SP and $A Q$ ) is directly observed by trained health workers; the other two daily doses of $\mathrm{AQ}$ alone are given to care givers to be administered at home, without supervision, which carries the risk of poor adherence $[19,20]$ and consequently sub-optimal drug levels in children already infected with malaria. Considering that both SP and AQ increase gametocyte carriage in $P$. falciparum malaria patients $[10,12]$ coupled with the risk of sub-optimal drug levels that could trigger gametocytogenesis [15], SMC may increase gametocyte carriage in asymptomatic malaria infected children, possibly resulting in onward transmission to the vector since it is administered during the rainy season when the malaria vector is abundant.

Here, the effect of SMC on post-treatment gametocyte carriage in asymptomatic $P$. falciparum infected children was assessed.

\section{Methods}

\section{Study setting}

This was a prospective study conducted during the 2017 and 2018 malaria transmission seasons in eastern Gambia. Malaria transmission in The Gambia, almost exclusively by $P$. falciparum, is highly seasonal, occurring mainly during the rainy season (July to October) and shortly after (November to December). Current control activities in the region include prompt diagnosis and treatment, vector control intervention such as indoor residual spraying (IRS) and insecticide-treated nets (ITN), and drug-based interventions such as intermittent preventive treatment during pregnancy (IPTp) and SMC, the latter implemented from 2014 onwards.

\section{Participants selection}

Potential participants were identified through the health and demographic surveillance system (HDSS) data base of villages with relatively high malaria prevalence $(\geq 20 \%)$ according to previous surveys [21]. These were children 24-59 months old and children 5-8 years old. The former represented the SMC group as they are eligible for SMC treatment; the latter were taken as comparison group as they do not receive SMC. Slightly older children were selected as a comparison group because all children within the SMC age group would receive the SMC treatment during the SMC campaigns. The assumption was that baseline gametocyte carriage would not vary between the two age groups [15, 22].

\section{Screening and enrolment}

At screening, information on medical history was collected and a clinical examination was performed. A dried blood spot (DBS) was collected via a finger prick for the detection of $P$. falciparum infection by polymerase chain reaction (PCR). Children with asymptomatic $P$. falciparum infection, without history or evidence of chronic illness and who have not received anti-malarial treatment in the previous 2 weeks were enrolled.

\section{Study visits before and after SMC treatment}

A visit was conducted by the study team close to the commencement of nation-wide SMC campaign (maximum 4 days prior). Demographic information and medical history, particularly any episode of clinical malaria or anti-malarial treatment since enrolment was collected. Axillary temperature and body weight were measured and recorded. Approximately $300 \mu \mathrm{L}$ of blood was collected by finger prick into an ethylene diamine tetra acetic acid (EDTA) microtainer tube for molecular detection and quantification of $P$. falciparum gametocytes. Haemoglobin was measured with a $\mathrm{HemoCue}^{\circledR}$ photometer (Ångelholm, Sweden). Parents and care givers 
were encouraged to participate in the upcoming SMC campaign and to ensure safe keeping of their SMC drug administration records.

The first SMC cycle was implemented by the Gambian National Malaria Control Programme. Briefly, a 3-day course of SP and AQ was administered by to all children aged 3 months to $<5$ years in the communities. The dose for the first day (SP and AQ) was directly observed by the health worker while that of second and third days (AQ alone) was administered by the caregiver. An SMC distribution card to document all administered doses for every child was issued to care givers.

Study participants were then re-visited by the study team approximately 2 weeks after the administration of the first SMC cycle. This time interval was chosen to allow for the emergence of gametocytes in the peripheral circulation [23]. History of any episode of clinical malaria or anti-malarial treatment since first study visit was collected. Axillary temperature was measured and children with fever (body temperature $>37.5^{\circ} \mathrm{C}$ ) were tested with a rapid diagnostic test (RDT) SD Bioline Malaria Ag P.F (Alere ${ }_{\mathrm{TM}}$ ) and if positive were considered clinical malaria cases and treated according to national guidelines. An additional blood sample $(300 \mu \mathrm{L})$ was collected by finger prick into an EDTA microtainer tube for molecular detection and quantification of P. falciparum gametocytes. For only the children in the SMC group, information on SMC adherence was collected as reported by care givers. In addition, each child's SMC administration card was reviewed and administered doses as documented was transcribed on the study case report form and used to assess participants' adherence to the treatment.

\section{Sample processing}

Blood samples collected in EDTA tubes during both study visits were stored in a cool box and transported to the laboratory within a maximum of $6 \mathrm{~h}$ after collection to maintain ribonucleic acid (RNA) stability [24]; $70 \mu \mathrm{l}$ of whole blood were immediately transferred into $350 \mu \mathrm{l}$ of RNAprotect Cell Reagent (Qigen, Hilden, Germany) and stored at $-70^{\circ} \mathrm{C}$. Laboratory staff were blinded to the study group of each sample during analysis. Each set of a participant's paired samples (i.e. sample for the same participant collected before and after SMC administration) were analysed for gametocytes in the same run to avoid variation in laboratory procedures.

Plasmodium falciparum diagnosis was performed at screening by PCR. Parasite deoxyribonucleic acid (DNA) was extracted from DBS using the QiaAmp DNA minikit (Qiagen, Germany). The var gene acidic terminal sequence (varATS) quantitative PCR was used to detect multi-copy genomic sequences of infections [25]. Briefly, genomic DNA of the parasite was amplified in $20 \mu \mathrm{l}$ reaction containing $1 \times$ Taqman mastermix (Life Technologies, United Kingdom) and run in CFX96 Touch $^{\mathrm{TM}}$ real-time PCR detection system (BioRad, United Kingdom). The starting quantity values of the parasite samples were estimated against laboratory grown $P$. falciparum 3D7 standard control (with medonic read of $3.74 \times 10^{6}$ erythrocytes/ $\mu$ l and thin film parasitaemia of 1197 parasites/ $\mu$ l of blood).

For $P$. falciparum gametocytes detection for the samples collected before and after SMC treatment, RNA was extracted using Qiagen's RNeasy ${ }^{\circledR}$ Mini kit according to the manufacturer's recommendations. Pfs 25 Quantitative Nucleic Acid Sequence-based Amplification (QT-NASBA) real-time PCR was performed on the extracted mRNAs using the following primers (forward primer: 5'-GACTGTAAATAAACCATGTGGAGA-3'; reverse primer $5^{\prime}$-AATTCTAATACGACTCACTAT AGGGAGAAGGCATTTACCGTTACCACAAGTTA$\left.3^{\prime}\right)$ and PCR conditions (pre-heat at $65^{\circ} \mathrm{C}$; incubate at $65{ }^{\circ} \mathrm{C}$ for 2 min then $41{ }^{\circ} \mathrm{C}$ for 2 min; a further $41{ }^{\circ} \mathrm{C}$ for $46 \mathrm{~s}$ incubation post enzyme introduction). Gametocytaemia was determined using fluorescence amplification time-points in correlation with the standard dilution series that was included in each run [22].

\section{Statistical analysis}

Sample size was calculated assuming baseline gametocyte prevalence of $50 \%$ in both the SMC and comparison groups [22]. A sample size of 116 evaluable participants in total, 58 per group, would have $80 \%$ power to detect a $25 \%$ difference (increase) in gametocyte prevalence between groups at the $5 \%$ significance level.

Statistical analysis was performed using STATA software version 16.0 (Stata Corp, College Station, Texas, USA). Descriptive statistics are presented for continuous variables (median (IQR)) and proportions for categorical variables; point estimates are presented with 95\% confidence intervals. Analysis was restricted to study participants who remained asymptomatic from enrolment to the time of post-treatment blood sampling. Gametocyte prevalence in children that received at least the first dose of SMC treatment (SP plus AQ) and in those that adhered to all 3-day doses of SMC treatment (SP plus AQ on day 1 and AQ alone on days 2 and 3) was each compared with gametocyte prevalence in the comparison group. Difference in prevalence between the two groups was assessed using the chi-squared test. Conditional logistic regression, conditional on individuals, was used to predict the odds of gametocyte carriage before and after treatment within each group. 


\section{Ethical consideration}

This study was approved by the Gambia Government/ MRC Joint Ethics Committee (SCC 1563). Children's parents or legal representative provided written informed consent prior to screening and study participation.

\section{Results}

A total of 1,567 children were screened for malaria infection at the beginning of two successive transmission seasons (2017 and 2018); 871 children aged 2 to $<5$ years (SMC group) and 696 aged 5 to 8 years (comparison group). Among these, 65 (7.5\%) children in the SMC group and $75(10.8 \%)$ in the comparison group had asymptomatic P. falciparum infection (Fig. 1). Baseline characteristics are as shown in Table 1. Gametocyte prevalence before SMC treatment was 10.7\% (7/65) and 13.3\% $(10 / 75)$ in the SMC and comparison groups respectively. Median interval (days) between receiving SMC treatment and blood sampling for assessment of post-treatment gametocytaemia was 13 days (IQR 12, 13). At 13 days post-treatment, there was no difference in gametocyte prevalence between children in the SMC group who received at least the first dose of SMC treatment (SP and AQ) $(9.4 \%, 5 / 53)$ and those in the comparison group $(12.7 \%, 9 / 71),(p=0.57)$. Although gametocyte prevalence was higher in children who adhered to all 3-day
Table 1 Baseline characteristics of study participants $(N=140)$

\begin{tabular}{|c|c|c|}
\hline \multirow[t]{2}{*}{ Characteristic } & \multicolumn{2}{|l|}{ Group } \\
\hline & $\mathrm{SMC}(n=65)$ & Comparison $(n=75)$ \\
\hline Gender (female), n (\%) & $31(47.7)$ & $35(46.7)$ \\
\hline Age (years), median (IQR) & $3.6(2.7-4.5)$ & $6.5(5.2-7.5)$ \\
\hline Weight (kg), median (IQR) & $12.8(11.0-14.2)$ & $18.0(15.5-20.6)$ \\
\hline $\begin{array}{l}\text { Haemoglobin }(\mathrm{g} / \mathrm{dL}) \text {, median } \\
\text { (IQR) }\end{array}$ & $10.9(9.9-12.0)$ & $11.1(10.0-12.2)$ \\
\hline $\begin{array}{l}\text { Asexual parasite density }(\text { per } \mu \mathrm{L}) \text {, } \\
\text { geometric mean }(95 \% \mathrm{Cl})\end{array}$ & $0.53(0.16-1.78)$ & $0.59(0.15-2.36)$ \\
\hline
\end{tabular}

doses of SMC treatment $(15.6 \%, 5 / 32)$, this was not significantly different with gametocyte prevalence of those in the comparison group $(p=0.68)$ (Table 2). Within the SMC group, gametocyte carriage tended to be lower after SMC treatment in children that received at least the first dose of SMC treatment, but this was not statistically significant (OR:0.6, 95\% CI: 0.14 to $2.51, p=0.48$ ). Likewise, there was no change in odds of gametocyte carriage after treatment among children that adhered to all 3-day doses of SMC treatment (OR 1.0, 95\% 0.20-4.95, $p=1.0$ ).

Adherence to SMC treatment and occurrence of vomiting after treatment was assessed in 90\% (48/53) of

\section{P. falciparum negative 36 Clinical malaria \\ 2 Chronic illness}

1427 Excluded

1567 Children Screened

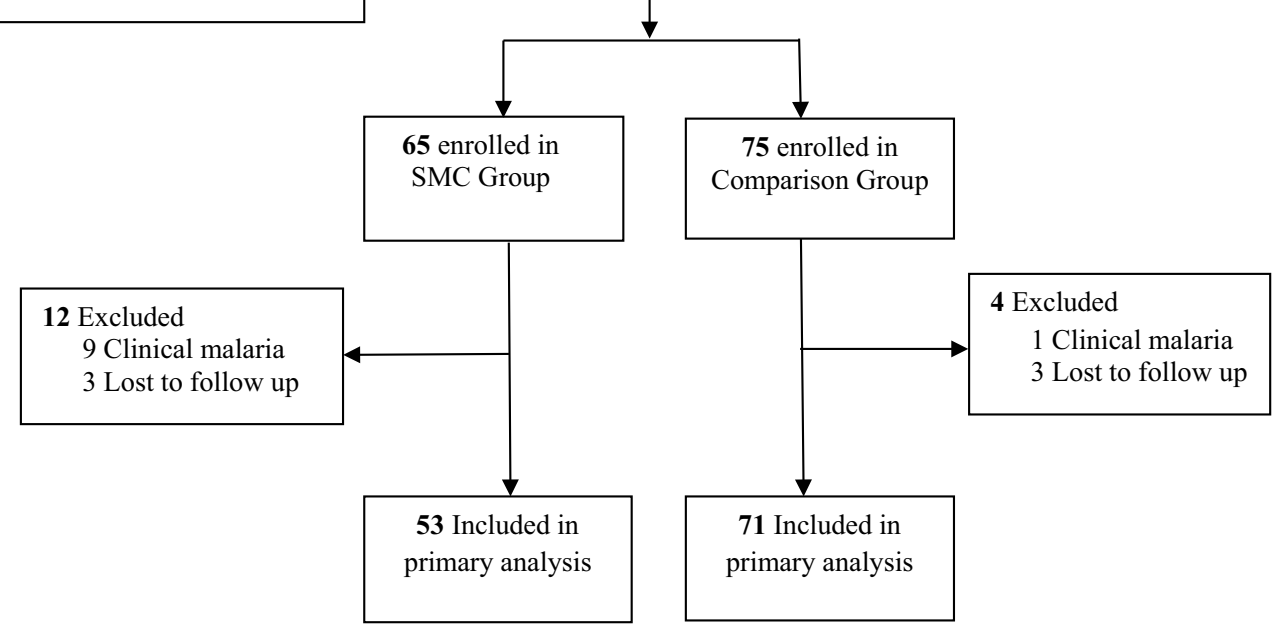

Fig. 1 Study Diagram 
Table 2 Differences in gametocyte carriage before and after SMC treatment

\begin{tabular}{|c|c|c|c|}
\hline \multirow[t]{2}{*}{ Measure } & \multicolumn{2}{|l|}{ Group } & \multirow[t]{2}{*}{ p value } \\
\hline & SMC & Comparison & \\
\hline Percentage of gametocyte carriers before SMC treatment & $7 / 65(10.7 \%)$ & 10/75 (13.3\%) & $0.64^{*}$ \\
\hline \multicolumn{4}{|l|}{ Percentage of gametocyte carriers after SMC treatment } \\
\hline Received at least first dose of treatment & $5 / 53(9.4 \%)$ & 9/71 (12.7\%) & $0.57^{*}$ \\
\hline Received all 3-day doses of treatment & $5 / 32(15.6 \%)$ & 9/71 (12.7\%) & $0.68^{*}$ \\
\hline \multicolumn{4}{|c|}{ Odds ratio comparing gametocyte carriage before vs after SMC treatment. OR (95\% CI) } \\
\hline Received at least first dose of treatment & $0.6(0.14-2.51)$ & & $0.48^{\infty}$ \\
\hline \multirow[t]{2}{*}{ Received all 3-day doses of treatment } & $1.0(0.20-4.95)$ & & $1.00^{\infty}$ \\
\hline & & $0.9(0.34-2.30)$ & $0.80^{\infty}$ \\
\hline
\end{tabular}

* Chi-squared test used to assess between-group difference in proportion of gametocyte carriers

$\infty$ Conditional logistic regression used to determine within-group odds of gametocyte carriage

children in the SMC group (Fig. 2). For all of them, the caregiver reported to have administered the remaining 2 days of treatment. When this information was crosschecked with the SMC drug administration card, only $68.7 \%(33 / 48)$ and $66.6 \%(32 / 48)$ had the doses of the second and third days documented on their cards, respectively. A total of $27.1 \%(13 / 48)$ of children were reported to have vomited the dose for the first day shortly after administration while $14.6 \%(7 / 48)$ and $12.5 \%(6 / 48)$ did so for the doses second and third days, respectively.

\section{Discussion}

$\mathrm{SP}$ and $\mathrm{AQ}$, the anti-malarial drugs deployed for the SMC intervention, are associated with marked increase in gametocytaemia when used to treat clinical cases of $P$. falciparum malaria $[10,12,14]$. Whether the administration of these drugs would increase prevalence of gametocytes among children with asymptomatic $P$. falciparum infections receiving SMC was investigated; no evidence of increased gametocyte prevalence was found.

Only a few studies have assessed the emergence of post-treatment gametocytes following treatment of asymptomatic $P$. falciparum infections and to our knowledge, no study has addressed this question in the context of the SMC. Dunyo et al. [26]. in a randomized controlled trial (RCT) assessed whether SP increased gametocyte carriage following treatment of asymptomatic $P$. falciparum carriers that had asexual parasite densities $>20$ parasites per micro litre but found no evidence of increased gametocyte prevalence or density. However, in that trial, microscopy was used to detect gametocytes and an increase in gametocytaemia may have been missed since emerging gametocytes typically circulate at or below the microscopic detection threshold [15]. In addition,

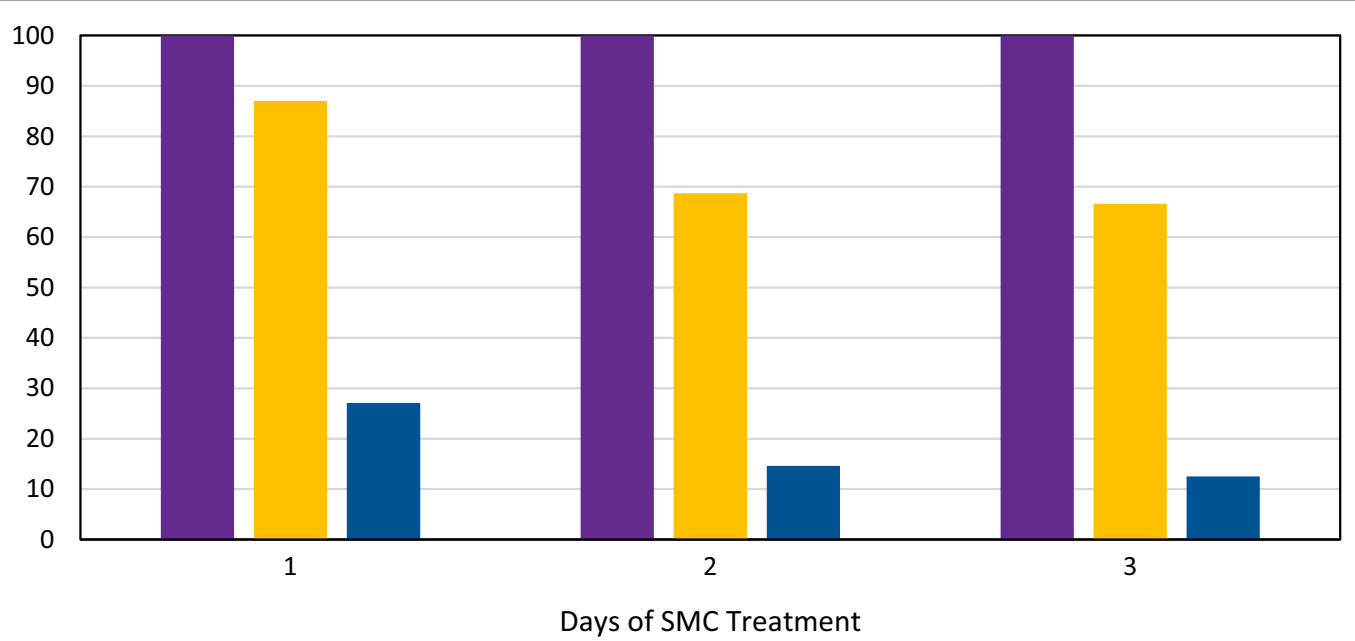

Fig. 2 Percentage of children that adhered to SMC treatment as reported by care giver (purple), as documented on the SMC distribution card (amber) and those that vomited administered dose shortly after administration (blue) 
considering the study by Dunyo et al. was a RCT, participants would have received the full treatment dose, with little or no risk of subcurative dosing that could trigger gametocyte production [27]. Conversely, sub-optimal dosage is probable in the context of standard SMC implementation wherein 2 out of the 3 daily doses are administered by caregivers $[19,20]$ and vomiting of administered doses shortly after administration is common [28]. However, even with the risk of sub-optimal dosaging and the use of sensitive molecular methods for detection of gametocytes in the current study, there was no evidence of increased gametocyte carriage following SMC in children that received either at least the first dose of SMC treatment or all 3-day doses of SMC treatment confirming findings by Dunyo et al. [26]. Of note however is that individuals enrolled in the current study had very low parasite densities (geometric mean $<1$ parasite/uL), representative of typical asymptomatic parasite carriers in low-endemic settings [29]. However, whilst the current study thus had much lower gametocyte prevalence than typically reported in infections with higher parasite densities [29], it has the advantage of including participants that reflect infected individuals among the actual target population of SMC.

In the current study population with low parasite density and low baseline gametocyte prevalence, sensitive molecular gametocyte detection methods did not reveal a significant effect of SMC (neither increase nor decrease) on gametocyte carriage shortly after treatment. Perhaps over longer-periods of follow-up, the clearance of asexual parasites, the precursors of gametocytes, may result in lower gametocyte carriage. This result could be enhanced if gametocyte-clearing drugs would be added to the SMC regimen, reducing gametocytes persistence after treatment that was also evident in the current study [18]. Such strategy might confer benefits for malaria transmission in the community.

Adherence to SMC administration as reported by caregivers was $100 \%$, similar to what has been reported from Mali [30]. However, when comparing reported adherence to the information on the SMC distribution card (documented adherence), the second and third doses were documented in only two thirds of study participants (Fig. 2), suggesting either caregivers' over-reporting of adherence during the interviews or incomplete recording. In a malaria chemoprevention trial in Uganda, drug levels were detectable in only $52 \%$ of cases despite caregivers having reported administering all assigned doses [20], suggesting over reporting of adherence. Similarly, a study on SMC uptake in Ghana concluded that up to $20 \%$ of children did not receive their second and third days treatments based on the number of tablets found at their homes during household visits [19]. Reported adherence by caregivers is, therefore, unreliable; possibly all SMC treatment doses should be directly observed by health workers or by trained village health workers, even though this approach could pose logistical challenges.

Vomiting occurred more often than expected [28], 27\% of children vomited the first treatment dose; although the percentage was lower for the second or third dose, this could be due to under-reporting by the caregivers. Low tolerability may lead to low adherence, resulting in sub-curative doses in malaria infected individuals, with potential risks of selecting for drug resistant parasites [31], emergence of post-treatment gametocytaemia [15] and onward malaria transmission [32]. Sweetened dispersible tablets resulted in higher tolerability in Senegal [33] and should perhaps be adopted as the standard formulation for SMC. Furthermore, provision should be made to compensate for vomited doses, particularly those administered by caregivers at home.

There are some limitations to this study that should be considered. Baseline gametocyte prevalence was lower than expected and this may have affected the power of the study to find a significant difference between SMC and comparison groups. However, there was little difference in gametocyte prevalence before and after treatment in the SMC group (10.7\% versus $9.4 \%)$ similar to what was observed in the control group (13.3\% versus $12.7 \%)$, suggesting that SMC is not associated with increased gametocyte carriage in the current study. Secondly, the fitness of gametocytes was not assessed as anti-malarial drugs may sterilize gametocytes before clearing them from circulation [34] since gametocyte transmissibility is ultimately more relevant for public health than gametocyte presence. Thirdly, drug concentrations were not measured and thus it is not possible to carry out a more detailed analysis on the effect of suboptimal dosing on post-treatment gametocytaemia. Finally, gametocytaemia was measured only at one time point after SMC treatment and this did not allow for plotting the dynamics of circulating gametocytes over a longer period.

\section{Conclusion}

In this low transmission setting, there is no evidence that SMC administered to asymptomatic P. falciparum infected children is associated with higher prevalence of gametocytes. This therefore argues against a possible increase in transmission after SMC campaigns. However, these findings cannot be generalizable to other settings since factors that could determine the emergence of posttreatment gametocytes, notably asexual parasite density [15] and drug resistance [35], vary across different transmission settings. As SMC is delivered to millions of children across different transmission settings of the Sahel, there is the need to investigate this question in other 


\section{settings ideally with direct assessments of transmission potential of gametocytes to mosquitoes.}

\begin{abstract}
Abbreviations
AQ: Amodiaquine; CQ: Chloroquine; DBS: Dried blood spot; DNA: Deoxyribonucleic acid; EDTA: Ethylene diamine tetraacetic acid; HDSS: Health and Demographic Surveillance System; IPTp: Intermittent preventive treatment during pregnancy; IRS: Indoor residual spraying; ITN: Insecticide treated nets; MRC: Medical Research Council; PQ: Piperaquine; PCR: Polymerase chain reaction; QT-NASBA: Quantitative nucleic acid sequence-based amplification; RCT: Randomized controlled trial; RDT: Rapid diagnostic test; RNA: Ribonucleic acid; SMC: seasonal malaria chemoprevention; SP: Sulfadoxine-pyrimethamine: varATS: var Gene acidic terminal sequence; WHO: World Health Organization.
\end{abstract}

\section{Acknowledgements}

We would like to thank the study volunteers who participated in this study We would want to express our gratitude to the Malaria Research Capacity Development Consortium (MARCAD) for providing the funding for this work. We would also like to thank the following staff from the MRCG at LSHTM who provided support during planning and conduct of the study: Davis Nwakanma, Muna Affara, Serign Ceesay, Musa Jawara, Lamin Gibba, Haddy Bittaye, Sainey Ceesay, Baboucarr Bittaye, Fatou Bah, Mavis Foster-Nyarko, Kebba Naban, Sampa Sanneh and the entire field team. JB received support from the UK MRC and the UK DFID (\#MR/R010161/1) under the MRC/DFID Concordat agreement and as part of the EDCTP2 programme supported by the European Union.

\section{Authors' contributions}

$A A, U D A$, and JA designed the study which was performed by $A A$ and $M B$. $\mathrm{AP}, \mathrm{BE}$, and $\mathrm{MN}$ performed molecular diagnostic analysis. JB, $\mathrm{AA}, \mathrm{UDA}$ and $T B$ analysed and interpreted the data and results. AA wrote the original draft manuscript which was critically reviewed and approved by all authors. All authors read and approved the final manuscript.

\section{Funding}

This work was supported through the DELTAS Africa Initiative grant DEL-15010. The DELTAS Africa Initiative is an independent funding scheme of the African Academy of Sciences (AAS)'s Alliance for Accelerating Excellence in Science in Africa (AESA) and supported by the New Partnership for Africa's Development Planning and Coordinating Agency (NEPAD Agency) with funding from the Wellcome Trust grant 107741/A/15/Z and the UK government. The views expressed in this publication are those of the author(s) and not necessarily those of AAS, NEPAD Agency, Wellcome Trust or the UK government'.

\section{Availability of data and materials}

The data used or analysed in this study are available from the corresponding author on reasonable request with approval from the Gambia Government/ MRC Joint Ethics Committee.

\section{Declarations}

\section{Ethics approval and consent to participate}

Ethics approvals were obtained from the Gambia Government/MRC Joint Ethics Committee (SCC 1563). Children's parents or legal representative provided written informed consent prior to screening and study participation.

\section{Consent for publication}

Consent for publication of study results and data which do not identify specific individuals was granted by each child's parent or legal representative when they enrolled for the study.

\section{Competing of interests}

All authors declare no competing interests.

\section{Author details}

${ }^{1}$ Disease Control and Elimination Theme, Medical Research Council Unit The Gambia At London, School of Hygiene and Tropical Medicine, P.O Box 273, Banjul, The Gambia. ${ }^{2}$ MRC Statistics and Epidemiology Group, London School of Hygiene and Tropical Medicine, Keppel Street, London WC1E 7HT, UK. ${ }^{3}$ Global Health Institute, University of Antwerp, Gouverneur Kinsbergencentrum, Campus Drie Eiken, Doornstraat 331, 2610 Wilrijk, Belgium. ${ }^{4}$ Department of Immunology and Infection, School of Hygiene and Tropical Medicine, Keppel Street, London WC1E 7HT, UK. ${ }^{5}$ Department of Medical Microbiology, Radboud University Medical Center, Geert Grooteplein 28, Microbiology 268, 6500 HB Nijmegen, The Netherlands.

Received: 16 November 2020 Accepted: 16 March 2021

Published online: 26 March 2021

\section{References}

1. WHO. World malaria report 2019. Geneva: World Health Organization; 2019.

2. Bhatt S, Weiss DJ, Cameron E, Bisanzio D, Mappin B, Dalrymple U, et al. The effect of malaria control on Plasmodium falciparum in Africa between 2000 and 2015. Nature. 2015;526:207-11.

3. WHO. Policy Recommendation: Seasonal Malaria Chemoprevention (SMC) for Plasmodium falciparum malaria control in highly seasonal transmission areas of the Sahel sub-region in Africa. Geneva: World Health Organization; 2012.

4. Lin JT, Saunders DL, Meshnick SR. The role of submicroscopic parasitemia in malaria transmission: what is the evidence? Trends Parasitol. 2014;30:183-90.

5. Bousema T, Okell L, Felger I, Drakeley C. Asymptomatic malaria infections: detectability, transmissibility and public health relevance. Nat Rev Microbiol. 2014:12:833-40.

6. Babiker HA, Abdel-Muhsin AM, Ranford-Cartwright LC, Satti G, Walliker D. Characteristics of Plasmodium falciparum parasites that survive the lengthy dry season in eastern Sudan where malaria transmission is markedly seasonal. Am J Trop Med Hyg. 1998:59:582-90.

7. Lindblade KA, Steinhardt L, Samuels A, Kachur SP, Slutsker L. The silent threat: asymptomatic parasitemia and malaria transmission. Expert Rev Anti Infect Ther. 2013;11:623-39.

8. WWARN Gametocyte Study Group. Gametocyte carriage in uncomplicated Plasmodium falciparum malaria following treatment with artemisinin combination therapy: a systematic review and meta-analysis of individual patient data. BMC Med. 2016;14:79.

9. Barnes KI, Little F, Mabuza A, Mngomezulu N, Govere J, Durrheim D, et al. Increased gametocytemia after treatment: an early parasitological indicator of emerging sulfadoxine-pyrimethamine resistance in falciparum malaria. J Infect Dis. 2008;197:1605-13.

10. Checchi F, Piola P, Kosack C, Ardizzoni E, Klarkowski D, Kwezi E, et al. Antimalarial efficacy of sulfadoxine-pyrimethamine, amodiaquine and a combination of chloroquine plus sulfadoxine-pyrimethamine in Bundi Bugyo, western Uganda. Trop Med Int Health. 2004;9:445-50.

11. Pasay CJ, Rockett R, Sekuloski S, Griffin P, Marquart L, Peatey C, et al. Piperaquine monotherapy of drug-susceptible Plasmodium falciparum infection results in rapid clearance of parasitemia but is followed by the appearance of gametocytemia. J Infect Dis. 2016;214:105-13.

12. Robert V, Awono-Ambene HP, Le Hesran JY, Trape JF. Gametocytemia and infectivity to mosquitoes of patients with uncomplicated Plasmodium falciparum malaria attacks treated with chloroquine or sulfadoxine plus pyrimethamine. Am JTrop Med Hyg. 2000;62:210-6.

13. Hallett RL, Dunyo S, Ord R, Jawara M, Pinder M, Randall A, et al. Chloroquine/sulphadoxine-pyrimethamine for gambian children with malaria: transmission to mosquitoes of multidrug-resistant Plasmodium falciparum. PLoS Clin Trials. 2006;1:e15.

14. Targett G, Drakeley C, Jawara M, von Seidlein L, Coleman R, Deen J, et al. Artesunate reduces but does not prevent posttreatment transmission of Plasmodium falciparum to Anopheles gambiae. J Infect Dis. 2001:183:1254-9.

15. Bousema T, Drakeley C. Epidemiology and infectivity of Plasmodium falciparum and Plasmodium vivax gametocytes in relation to malaria control and elimination. Clin Microbiol Rev. 2011;24:377-410.

16. Nwakanma D, Kheir A, Sowa M, Dunyo S, Jawara M, Pinder M, et al. High gametocyte complexity and mosquito infectivity of Plasmodium falciparum in the Gambia. Int J Parasitol. 2008;38:219-27. 
17. Darling ST. Studies in relation to malaria. Washington Government Printing Office. 1910

18. Dicko A, Roh ME, Diawara H, Mahamar A, Soumare HM, Lanke K, et al. Efficacy and safety of primaquine and methylene blue for prevention of Plasmodium falciparum transmission in Mali: a phase 2, single-blind randomised controlled trial. Lancet Infect Dis. 2018;18:627-39.

19. Antwi GD, Bates LA, King R, Mahama PR, Tagbor H, Cairns M, et al. Facilitators and barriers to uptake of an extended seasonal malaria chemoprevention programme in Ghana: a qualitative study of caregivers and community health workers. PLoS ONE. 2016;11:e0166951.

20. Bigira V, Kapisi J, Clark TD, Kinara S, Mwangwa F, Muhindo MK, et al. Protective efficacy and safety of three antimalarial regimens for the prevention of malaria in young Ugandan children: a randomized controlled trial. PLoS Med. 2014;11:e1001689.

21. Mwesigwa J, Okebe J, Affara M, Di Tanna GL, Nwakanma D, Janha O, et al. Ongoing malaria transmission in The Gambia despite high coverage of control interventions: a nationwide cross-sectional survey. Malar J. 2015;14:314.

22. Okebe J, Bousema T, Affara M, Di Tanna GL, Dabira E, Gaye A, et al. The gametocytocidal efficacy of different single doses of primaquine with dihydroartemisinin-piperaquine in asymptomatic parasite carriers in The Gambia: a randomized controlled trial. EBioMedicine. 2016;13:348-55.

23. Reuling IJ, van de Schans LA, Coffeng LE, Lanke K, Meerstein-Kessel L, Graumans W, et al. A randomized feasibility trial comparing four antimalarial drug regimens to induce Plasmodium falciparum gametocytemia in the controlled human malaria infection model. eLife. 2018;7:e31549.

24. Waltmann A, Karl S, Chiu C, Mueller I. Limited degradation of the Plasmodium falciparum gametocyte marker pfs 25 mRNA exposed to tropical temperatures: considerations for malaria transmission field studies. Am J Trop Med Hyg. 2016;94:886-9.

25. Hofmann N, Mwingira F, Shekalaghe S, Robinson LJ, Mueller I, Felger I. Ultra-sensitive detection of Plasmodium falciparum by amplification of multi-copy subtelomeric targets. PLoS Med. 2015;12:e1001788.

26. Dunyo S, Milligan P, Edwards T, Sutherland C, Targett G, Pinder M. Gametocytaemia after drug treatment of asymptomatic Plasmodium falciparum. PLoS Clin Trials. 2006;1:e20
27. Buckling A, Ranford-Cartwright LC, Miles A, Read AF. Chloroquine increases Plasmodium falciparum gametocytogenesis in vitro. Parasitology. 1999;118:339-46.

28. Ndiaye JL, Cissé B, Ba EH, Gomis JF, Ndour CT, Molez JF, et al. Safety of seasonal malaria chemoprevention (SMC) with sulfadoxine-pyrimethamine plus amodiaquine when delivered to children under 10 years of age by district health services in Senegal: results from a stepped-wedge cluster randomized trial. PLoS ONE. 2016;11:e0162563.

29. Slater HC, Ross A, Felger I, Hofmann NE, Robinson L, Cook J, et al. The temporal dynamics and infectiousness of subpatent Plasmodium falciparum infections in relation to parasite density. Nat Commun. 2019;10:1433.

30. Diawara F, Steinhardt LC, Mahamar A, Traore T, Kone DT, Diawara H, et al. Measuring the impact of seasonal malaria chemoprevention as part of routine malaria control in Kita, Mali. Malar J. 2017:16:325.

31. White NJ. Antimalarial drug resistance. J Clin Invest. 2004;113:1084-92.

32. Challenger JD, Gonçalves BP, Bradley J, Bruxvoort K, Tiono AB, Drakeley $C$, et al. How delayed and non-adherent treatment contribute to onward transmission of malaria: a modelling study. BMJ Glob Health. 2019;4:e001856.

33. Cissé B, Ba EH, Sokhna C, Gomis JF, Dial Y, et al. Effectiveness of seasonal malaria chemoprevention in children under ten years of age in Senegal: a stepped-wedge cluster-randomised trial. PLoS Med. 2016;13:1002175.

34. Alkema M, Reuling IJ, de Jong GM, Lanke K, Coffeng LE, van Gemert GJ, et al. A randomized clinical trial to compare $P$. falciparum gametocytaemia and infectivity following blood-stage or mosquito bite induced controlled malaria infection. J Infect Dis. 2020; jiaa157 (online ahead of print).

35. White NJ. The role of anti-malarial drugs in eliminating malaria. Malar J. 2008;7:S8.

\section{Publisher's Note}

Springer Nature remains neutral with regard to jurisdictional claims in published maps and institutional affiliations.
Ready to submit your research? Choose BMC and benefit from:

- fast, convenient online submission

- thorough peer review by experienced researchers in your field

- rapid publication on acceptance

- support for research data, including large and complex data types

- gold Open Access which fosters wider collaboration and increased citations

- maximum visibility for your research: over $100 \mathrm{M}$ website views per year

At BMC, research is always in progress.

Learn more biomedcentral.com/submissions 